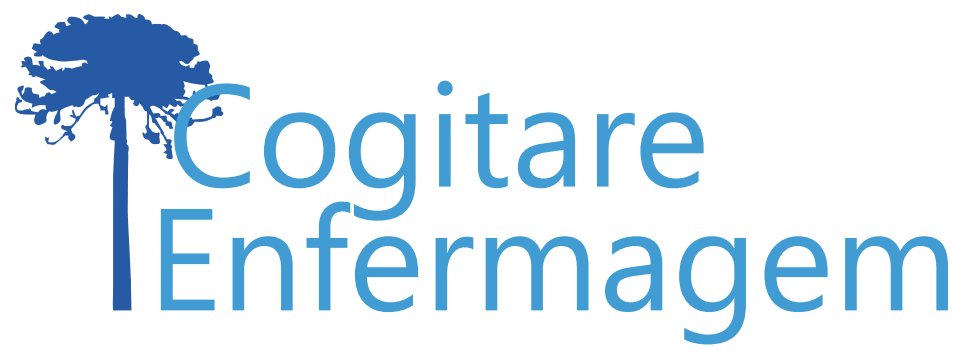

\title{
PERFIL SOCIODEMOGRÁFICO E CLÍNICO DE MULHERES COM CÂNCER NO TRATO GENITAL SUBMETIDAS À RADIOTERAPIA
}

\author{
Anizelle Aline Lopes da Silva1, Luciana Martins da Rosa², Soraia Dornelles Schoeller ${ }^{3}$, Vera \\ Radünz ${ }^{4}$, Maria Manuela Martins ${ }^{5}$, Henriqueta llda Verganista Martins Fernandes ${ }^{6}$, Érica \\ Bernardes Duarte ${ }^{7}$
}

\section{RESUMO}

Objetivo: caracterizar o perfil sociodemográfico e clínico de mulheres com câncer no trato genital submetidas à radioterapia no Centro de Pesquisas Oncológicas entre 2010 e 2014.

Método: estudo ecológico realizado em 880 arquivos do Registro Hospitalar de Câncer. A análise ocorreu por estatística descritiva, com cálculo de taxa de prevalência.

Resultados: a maior incidência, 204 casos (23,18\%) ocorreu na faixa etária dos 40-49 anos; com maior taxa de prevalência dos 60 a 69 anos, 165 casos $(82,28 \%)$ para cada 100.000 mulheres. Estádio III com 315 casos (35,8\%), topografia colo do útero com 695 casos $(78,97 \%)$ e respectivas taxas de prevalências $12,97 \%, 28,61 \%$. Dos casos de câncer do colo do útero, 274 (39,77\%) foram procedentes da Grande Florianópolis e 99 (14,37\%) da macrorregião Sul.

Conclusão: reafirma-se a magnitude do câncer do colo do útero, a necessidade das políticas públicas para prevenção da doença e do diagnóstico precoce das lesões pré-neoplásicas e/ou neoplásicas.

DESCRITORES: Neoplasias dos genitais femininos; Neoplasias do colo do útero; Radioterapia; Perfil de saúde; Registros hospitalares.

\section{COMO REFERENCIAR ESTE ARTIGO:}

Silva AAL, Rosa LM, Schoeller SD, Radünz V, Martins MM, Martins HIV, et al. Perfil sociodemográfico e clínico de mulheres com câncer no trato genital submetidas à radioterapia. Cogitare enferm. [Internet]. 2019 [acesso em "colocar data de acesso, dia, mês abreviado e ano"]; 24. Disponível em: http://dx.doi.org/10.5380/ ce.v24i 0.58467.

\section{(c) (1)}

Este obra está licenciado com uma Licença Creative Commons Atribuição 4.0 Internacional.

${ }^{1}$ Enfermeira. Universidade Federal de Santa Catarina. Laboratório de Pesquisa Cuidando \& Confortando. Florianópolis, SC, Brasil. 1

${ }^{2}$ Enfermeira. Doutora em Enfermagem. Docente de Enfermagem e de Pós-Graduação em Enfermagem da Universidade Federal de Santa Catarina. Florianópolis, SC, Brasil. 9

${ }^{3}$ Enfermeira. Doutora em Enfermagem. Docente de Enfermagem e de Pós-Graduação em Enfermagem da Universidade Federal de Santa Catarina. Florianópolis, SC, Brasil. 1

${ }^{4}$ Enfermeira. Docente de Enfermagem da Universidade Federal de Santa Catarina. Florianópolis, SC, Brasil. $\odot$

${ }^{5}$ Enfermeira. Doutora em Enfermagem. Professora. Núcleo de Investigação de Enfermagem de Família (NIEF). Grupo de Investigação - NursID: Inovação e Desenvolvimento em Enfermagem - CINTESIS - Center for Health Technology and Services Research - FMUP. Porto, Portugal. (

${ }^{6}$ Enfermeira. Doutora em Enfermagem. Professora. Mestre em Ciência de Enfermagem e PhD em Educação. Escola Superior de Enfermagem do Porto. Membro do CINTESIS. Porto, Portugal. $\bigcirc$

${ }^{7}$ Enfermeira. Universidade Federal de Santa Catarina. Florianópolis, SC, Brasil. 


\title{
SOCIODEMOGRAPHIC AND CLINICAL PROFILE OF WOMEN WITH CANCER IN THE GENITAL TRACT WHO UNDERWENT RADIATION THERAPY
}

\begin{abstract}
Objective: To characterize the sociodemographic and clinical profile of women with cancer in the genital tract who underwent radiation therapy at the Centro de Pesquisas Oncológicas between 2010 and 2014.

Method: Ecological study of 880 files of the Hospital-based Cancer Registry. Descriptive statistics, with calculation of the prevalence rate was used in the analysis.

Results: The higher incidence, 204 cases (23.18\%) occurred in the age group of 40-49 years; with a higher prevalence rate from 60 to 69 years, 165 cases (82.28\%) for every 100,000 women. Stage III with 315 cases (35.8\%), cervical topography with 695 cases (78.97\%) and respective prevalence rates $12.97 \%, 28.61 \%$. Of the total cases of cervical cancer, $274(39.77 \%)$ were from Grande Florianópolis (Santa Catarina, Brazil) and 99 (14.37\%) from the southern macroregion. Conclusion: The importance of cervical cancer, the need for public policies for disease prevention and early diagnosis of pre-neoplastic and/or cancer lesions are reaffirmed here.
\end{abstract}

DESCRIPTORS: Cancer of female genital organs; Cervical cancers; Radiotherapy; Health profile; Hospital records.

\section{PERFIL SOCIAL DEMOGRÁFICO Y CLÍNICO DE MUJERES CON CÁNCER EN EL TRATO GENITAL SOMETIDAS A LA RADIOTERAPIA}

\begin{abstract}
RESUMEN
Objetivo: caracterizar el perfil social demográfico y clínico de mujeres con cáncer en el trato genital sometidas a la radioterapia en el Centro de Pesquisas oncológicas entre 2010 y 2014. Método: estudio ecológico que se realizó por medio de 880 archivos del Registro Hospitalario de Cáncer. El análisis se hizo por estadística descriptiva, con cálculo de taja de prevalencia. Resultados: la mayor incidencia, 204 casos (23,18\%), ocurrió en la franja etaria de los 40 49 años; con mayor taja de prevalencia de los 60 a 69 años, 165 casos (82,28\%) para cada 100.000 mujeres. Estadio III con 315 casos (35,8\%), topografía de cuello del útero con 695 casos $(78,97 \%)$ y respectivas tajas de prevalencias $12,97 \%, 28,61 \%$. De los casos de cáncer del cuello de útero, $274(39,77 \%)$ ocurrieron en la Grande Florianópolis (Santa Catarina, Brasil) y $99(14,37 \%)$ en la macro región Sur.

Conclusión: se reafirman la magnitud del cáncer de cuello del útero, la necesidad de las políticas públicas para prevención de la enfermedad y el diagnóstico precoz de las lesiones pre neoplásicas y/o neoplásicas.
\end{abstract}

DESCRIPTORES: Neoplasias de los genitales femeninos; Neoplasias del cuello del útero; Radioterapia; Perfil de salud; Registros hospitalarios. 
Os cânceres no trato genital feminino apresentam elevada incidência, destacando-se o câncer do colo do útero. Essas neoplasias trazem consequências à saúde da mulher, pois comprometem o físico, o emocional, a feminilidade e a sexualidade da mulher. No mundo e no Brasil, a incidência dos cânceres do trato genital mais incidentes são, respectivamente, de 569.847 e 16.370 casos novos no colo do útero, 319.605 e 6.600 casos novos no corpo do útero, 238.719 e 6.150 casos novos no ovário ${ }^{(1-2)}$.

No tratamento dos cânceres no trato genital, é comum o uso da radioterapia, na modalidade teleterapia e braquiterapia. Na teleterapia ou radioterapia externa, existe distância física entre o paciente e a fonte da radiação ${ }^{(3)}$. $\mathrm{Na}$ braquiterapia, a radiação é administrada próxima ao tumor. Esta modalidade tem bom potencial devido a sua alta característica de gradiente de dose, e ainda permite a preservação do tecido normal adjacente. No câncer de colo do útero, o mais comum entre as mulheres, a braquiterapia desempenha papel importante no controle local da doença ${ }^{(4)}$. Neste estudo, as mulheres em radioterapia foram submetidas, à teleterapia e/ou à braquiterapia.

Em Santa Catarina, o Centro de Pesquisas Oncológicas (CEPON), uma instituição referência para o Estado, atendeu entre os anos 2010 e 2014 (único período de dados registrados no Registro Hospitalar de Câncer) 887 mulheres com câncer no trato genital que necessitaram de tratamento com radioterapia. Conforme a gestão dos atendimentos no Sistema Único de Saúde em Santa Catarina, o CEPON atende todos os casos de cânceres da macrorregião da grande Florianópolis e todos os outros casos das outras macrorregiões do Estado, encaminhados à instituição para Tratamento Fora do Domicílio (TFD). O TFD permite o atendimento de saúde fora do município de origem por falta de condições técnicas.

Destaca-se que, entre 2006 e 2016, o CEPON era a única instituição pública estadual a ofertar a terapêutica braquiterápica para o controle dos cânceres no trato genital. Assim, todas as mulheres catarinenses que necessitaram de braquiterapia entre o ano 2010 e o ano 2014 (recorte deste estudo) foram encaminhadas para o CEPON, porém o diagnóstico e as terapêuticas iniciais para o controle das doenças foram realizados nas macrorregiões de procedência de cada mulher.

Ressalta-se que o CEPON possui Registro Hospitalar de Câncer (RHC) e os dados relacionados às mulheres acometidas por cânceres no trato genital, submetidas à radioterapia, ainda não foram analisados. Os RHCs (fonte dos dados analisados deste estudo) registram teleterapia e braquiterapia com o mesmo símbolo: RT.

O RHC configura um banco de dados informatizado e público, que disponibiliza de forma sistemática e contínua informações de pacientes com diagnóstico confirmado de câncer ${ }^{(5)}$. Por meio dos dados fornecidos pelo RHC, é possível obter-se informações que possam auxiliar no planejamento administrativo da atenção oncológica às mulheres com câncer no trato genital, submetidas à radioterapia no CEPON, pois a análise desses dados poderá melhorar a gestão do serviço de saúde da instituição(b).

Portanto, o objetivo firmado para este estudo foi caracterizar o perfil sociodemográfico e clínico de mulheres com câncer no trato genital, submetidas à radioterapia no Centro de Pesquisas Oncológicas entre 2010 e 2014.

\section{MÉTODO}

Trata-se de um estudo ecológico, com inclusão no estudo dos registros de todas as mulheres com neoplasias malignas no trato genital, submetidas à radioterapia no recorte de tempo entre 2010 e 2014, com exclusão de mulheres com sarcomas e neoplasias oncohematológicas no trato genital. 
As variáveis do estudo foram: idade (faixa etária $\leq 19$ anos; 20-29; 30-39; 40-49; 50-59; 60-69;70 anos ou mais); raça/cor; estado conjugal; grau de instrução; procedência por macrorregião de Santa Catarina (definidas pelo Governo do Estado, conforme o Plano Diretor de Regionalização de 2008); morfologia (adenocarcinoma, carcinoma ou outros); estadiamento clínico, topografia (local da neoplasia, segundo Classificação Internacional de Doenças para Oncologia (CID-0): vulva (C51.9); vagina (C52.9); colo do útero (C53.0; C53.1; C53.8; C53.9); corpo do útero (C54.1; C54.9) e ovário (C56.9). Para facilitar os cálculos estatísticos os CIDs: C53.0; C53.1; C53.8; C53.9 foram agrupados na topografia colo do útero e os CID: C54.1; C54.9 foram agrupados na topografia corpo do útero.

Para coleta dos dados, solicitou-se os dados referentes às variáveis do estudo ao RHC/CEPON em março de 2017.

Após os dados serem disponibilizados pelo $\mathrm{RHC}$, foram salvos em planilhas construídas no programa Excel ${ }^{\circledR}$ 2016. As variáveis foram codificadas e submetidas à estatística descritiva. As variáveis estadiamento e faixa etária; diagnóstico e faixa etária; macrorregião e faixa etária; macrorregião e diagnóstico foram correlacionadas. As variáveis faixa etária, estadiamento e topografia das neoplasias foram submetidas ao cálculo de taxa de prevalência dos casos. Para tanto, foi utilizada a estatística descritiva e teste de correlação não paramétrico.

Em relação aos aspectos éticos, destaca-se que o estudo foi aprovado por Comitê de Ética em Pesquisa, sob o número do parecer 1.948.795, realizado no banco de dados do Registro Hospitalar de Câncer do Centro de Pesquisas Oncológicas (RHC/CEPON).

\section{RESULTADOS}

Dos dados disponibilizados pelo RHC/CEPON, verificou-se o atendimento, no período 2010-2014, de 887 mulheres com câncer no trato genital submetidas à radioterapia. Destes registros, sete foram excluídos por estarem relacionados ao diagnóstico de sarcoma e ao diagnóstico de linfoma (critério de exclusão). Assim, a população do estudo foi de 880 registros (100\%). O número de atendimentos por ano foi de 188 casos $(21,36 \%)$ em 2010; 239 casos (27,16\%) em 2011; 130 casos (14,77\%) em 2012; 73 casos (8,3\%) em 2013; 250 casos (28,41\%) em 2014.

A faixa etária dos 40-49 anos com 204 casos (23,18\%) e dos 50-59 anos com 201 casos $(22,84 \%)$ foram as faixas etárias com maior incidência. A média das idades foi de 51,53 anos, sendo a idade mínima de 14 anos, a máxima de 90 anos e a amplitude de 76 anos. A faixa etária com maior taxa de prevalência foi a de 60 a 69 anos, com 82,2 casos para cada 100.000 mulheres. Porém, somente até 29 anos a taxa de prevalência se manteve abaixo das 10/0000 mulheres, aumentando após a idade de 30 anos (Tabela 1).

Tabela 1 - Número de mulheres com diagnóstico de câncer no trato genital, atendidas no Centro de Pesquisas Oncológicas entre 2010-2014, e taxa de prevalência dos casos segundo faixa etária. Florianópolis, SC, Brasil, 2017 (continua)

\begin{tabular}{lccc} 
Idade & $\mathbf{N}$ & $\%$ & $\begin{array}{c}\text { Taxa de } \\
\text { prevalência/1000 } \\
\text { mulheres }\end{array}$ \\
\hline$\leq 19$ anos & 2 & 0,23 & 0,74 \\
\hline 20 a 29 anos & 48 & 5,45 & 8,59 \\
\hline 30 a 39 anos & 154 & 17,5 & 31,34 \\
\hline 40 a 49 anos & 204 & 23,18 & 44,42 \\
\hline
\end{tabular}




\begin{tabular}{lccc}
\hline 50 a 59 anos & 201 & 22,84 & 59,03 \\
\hline 60 a 69 & 165 & 18,75 & 82,28 \\
\hline 70 ou mais & 106 & 12,04 & 64,5 \\
\hline TOTAL & 880 & 100 & 36,22
\end{tabular}

O nível de escolaridade mais frequente entre as mulheres foi o fundamental incompleto e completo com $558(63,41 \%)$; seguido do ensino médio incompleto e completo com 147 $(16,70 \%)$; ensino superior incompleto e completo, com 55 (6,25\%); analfabeta com 34 $(3,86 \%)$ e 86 mulheres $(9,77 \%)$ não tinham informações sobre a escolaridade.

Em relação à cor ou raça das mulheres, foram identificadas 829 (94,20\%) brancas, $24(2,73 \%)$ negras, $21(2,39 \%)$ pardas e uma $(0,11 \%)$ amarela. Não foram encontradas informações de cinco $(0,57 \%)$ mulheres quanto à cor da pele.

A Tabela 2 correlaciona o estado conjugal com a idade das mulheres, sendo a maioria casada 431 (48,98\%) e com idade média de 51 anos.

Tabela 2 - Estado conjugal de mulheres com diagnóstico de câncer no trato genital, atendidas no Centro de Pesquisas Oncológicas entre 2010-2014, segundo idade média e percentual. Florianópolis, SC, Brasil, 2017

\begin{tabular}{lccc} 
Estado conjugal & N & Idade média & $\%$ \\
\hline Casada & 431 & 51,47 & 48,98 \\
\hline Solteira & 159 & 42,77 & 18,07 \\
\hline Viúva & 118 & 65,41 & 13,41 \\
\hline União consensual & 47 & 41,73 & 5,34 \\
\hline Separada judicialmente & 93 & 53,65 & 10,57 \\
\hline Sem informação & 32 & -- & 3,64 \\
\hline Total & 880 & -- & 100
\end{tabular}

Dos 880 registros incluídos no estudo, 36 (4,09\%) não registravam o estadiamento das doenças (sem informação). O estádio III com 315 casos $(35,8 \%)$ e o II com 293 casos (33,30\%) apresentaram os maiores achados. Correlacionando o estádio e faixa etária, os maiores valores encontrados ocorreram nos estádios III e II e faixas etárias dos 41-50 anos e 51-60 anos, conforme dados apresentados na Tabela 3. 
Tabela 3 - Frequência dos estadiamento de mulheres com câncer no trato genital, atendidas no Centro de Pesquisas Oncológicas entre 2010-2014, segundo faixa etária, idade média e taxa deprevalência dos casos. Florianópolis, SC, Brasil, 2017

\begin{tabular}{|c|c|c|c|c|c|c|c|c|c|c|c|c|c|c|}
\hline Estadiamento & 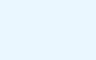 & 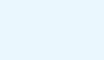 & II & & II & & & & in $\mathrm{s}$ & & Sen & & & \\
\hline Faixa etária & $\mathrm{N}$ & $\%$ & $\mathrm{~N}$ & $\%$ & $\mathrm{~N}$ & $\%$ & $\mathrm{~N}$ & $\%$ & $\mathrm{~N}$ & $\%$ & $\mathrm{~N}$ & $\%$ & $\mathrm{~N}$ & $\%$ \\
\hline$<$ de 19 anos & 1 & 0,1 & 0 & 0 & 1 & 0,1 & 0 & 0 & 0 & 0 & 0 & 0 & 2 & 0,2 \\
\hline 20 a 29 anos & 7 & 0,8 & 14 & 1,5 & 20 & 2,2 & 5 & 0,5 & 0 & 0 & 2 & 0,2 & 48 & 5,4 \\
\hline 30 a 39 anos & 23 & 2,6 & 55 & 6,2 & 58 & 6,5 & 14 & 1,5 & 3 & 0,3 & 1 & 0,1 & 154 & 17,5 \\
\hline 40 a 49 anos & 29 & 3,3 & 72 & 8,1 & 82 & 9,3 & 13 & 1,4 & 0 & 0 & 8 & 0,9 & 204 & 23,1 \\
\hline 50 a 59 anos & 39 & 4,4 & 76 & 8,6 & 65 & 7,3 & 11 & 1,2 & 2 & 0,2 & 8 & 0,9 & 201 & 22,8 \\
\hline 60 a 69 & 45 & 5,1 & 53 & 6 & 54 & 6,1 & 5 & 0,5 & 2 & 0,2 & 6 & 0,6 & 165 & 18,7 \\
\hline 70 e mais & 29 & 3,3 & 23 & 2,6 & 35 & 3,9 & 8 & 0,9 & 2 & 0,2 & 9 & 1 & 106 & 12 \\
\hline Total & 173 & 19,6 & 293 & 33,3 & 315 & 35,8 & 56 & 6,3 & 7 & 0,8 & 36 & 4 & 880 & 100 \\
\hline $\begin{array}{l}\text { Idade média em } \\
\text { anos }\end{array}$ & 61 & -- & 60,03 & -- & 50,51 & -- & 50,3 & -- & 61,5 & -- & 48,1 & -- & & -- \\
\hline $\begin{array}{l}\text { Tx prevalência/ } \\
100.000 \text { mulheres }\end{array}$ & 7,12 & & 12,06 & & 12,97 & & 2,3 & & 1,48 & & 0,29 & -- & 36,2 & -- \\
\hline
\end{tabular}

A Tabela 4 apresenta os percentuais por topografia (localização anatômica da neoplasia maligna). O câncer de colo do útero (incluindo colo do útero, endocérvix, exocérvix e lesão sobreposta do colo do útero) foi o mais prevalente, com 695 casos $(78,97 \%)$, seguido do corpo do útero, com 166 casos $(18,87 \%)$, sendo que, do total destes, a topografia endométrio foi a mais acometida no corpo do útero, com 159 casos $(18,06 \%)$.

Tabela 4 - Topografia das neoplasias no trato genital de mulheres submetidas à radioterapia, no Centro de Pesquisas Oncológicas entre 2010-2014, e taxa de prevalência dos casos. Florianópolis, SC, Brasil, 2017

\begin{tabular}{lccc} 
Topografia & $\mathbf{N}$ & $\%$ & $\begin{array}{c}\text { Taxa de prevalência/100.000 } \\
\text { mulheres entre 14 a mais de } \\
\mathbf{7 0} \text { anos }\end{array}$ \\
\hline Colo do útero & & 78,97 & 28,61 \\
\hline Corpo do útero & 695 & 18,87 & 6,83 \\
\hline Vulva & 166 & 0,91 & 33 \\
\hline Vagina & 8 & 0,57 & 0,21 \\
\hline Ovário & 5 & 0,68 & 0,25 \\
\hline Total & 6 & 100 & 36,22
\end{tabular}

A Tabela 5 mostra o percentual por topografia investigada. Considerando que a maioria das mulheres foi diagnosticada com câncer do colo do útero e do corpo do útero, estas topografias foram destacadas e as outras neoplasias foram agrupadas. A topografia foi correlacionada com as macrorregiões do Estado de Santa Catarina. Assim, observa-se 
que o maior atendimento das mulheres com câncer no colo do útero refere-se às mulheres residentes na Grande Florianópolis com 274 casos (39,77\%), seguida da macrorregião Sul com 99 casos (14,37\%), Nordeste com 85 casos (12,34\%) e Extremo Oeste com 84 casos $(12,19 \%)$. Para mulheres com câncer do corpo do útero, o número maior de atendimento por procedência das macrorregiões assemelha-se ao já apontado. Entretanto, 511 casos $(58,07 \%)$ atendidos no CEPON, de todas as topografias, não foram provenientes da Grande Florianópolis. A totalidade dos resultados é apresentada na Tabela 5.

Tabela 5 - Topografia das neoplasias do trato genital de mulheres submetidas à radioterapia, no Centro de Pesquisa Oncológicas entre 2010-2014, segundo macrorregião do Estado de Santa Catarina. Florianópolis, SC, Brasil, 2017

\begin{tabular}{|c|c|c|c|c|c|c|c|c|c|c|c|}
\hline \multirow[t]{2}{*}{ Macrorregião } & \multicolumn{3}{|c|}{$\begin{array}{c}\text { Colo do útero e } \\
\text { endocervix }\end{array}$} & \multicolumn{3}{|c|}{ Corpo do útero } & \multicolumn{3}{|c|}{ Outros } & \multicolumn{2}{|c|}{ Total } \\
\hline & $\mathbf{N}$ & $\begin{array}{c}\% \\
\text { Macro }\end{array}$ & $\begin{array}{l}\% \\
\text { SC }\end{array}$ & $\mathbf{N}$ & $\begin{array}{c}\% \\
\text { Macro }\end{array}$ & $\begin{array}{l}\% \\
\text { sC }\end{array}$ & $\mathbf{N}$ & $\begin{array}{c}\% \\
\text { Macro }\end{array}$ & $\begin{array}{l}\% \\
\text { SC }\end{array}$ & $\mathbf{N}$ & $\begin{array}{l}\% \\
\text { SC }\end{array}$ \\
\hline Sul & 99 & 14,37 & 11,25 & 19 & 11,59 & 2,16 & 3 & 11,11 & 0,34 & 121 & 13,75 \\
\hline $\begin{array}{l}\text { Grande } \\
\text { Florianópolis }\end{array}$ & 274 & 39,77 & 31,14 & 76 & 46,34 & 8,64 & 19 & 70,37 & 2,16 & 369 & 41,93 \\
\hline Foz do Rio Itajaí & 2 & 0,29 & 0,23 & 0 & 0 & 0 & 1 & 3,7 & 0,11 & 3 & 0,34 \\
\hline Nordeste & 85 & 12,34 & 9,66 & 10 & 6,1 & 1,14 & 1 & 3,7 & 0,11 & 96 & 10,91 \\
\hline Planalto Norte & 23 & 3,34 & 2,61 & 9 & 5,49 & 1,02 & 0 & 0 & 0 & 32 & 3,64 \\
\hline Vale do Itajaí & 6 & 0,87 & 0,68 & 3 & 1,83 & 0,34 & 1 & 3,7 & 0,11 & 10 & 1,14 \\
\hline Planalto Serrano & 43 & 6,24 & 4,89 & 10 & 6,1 & 1,14 & 0 & 0 & 0 & 53 & 6,02 \\
\hline Meio Oeste & 73 & 10,6 & 8,3 & 19 & 11,59 & 2,16 & 1 & 3,7 & 0,11 & 93 & 10,57 \\
\hline Extremo Oeste & 84 & 12,19 & 9,55 & 18 & 10,98 & 2,05 & 1 & 3,7 & 0,11 & 103 & 11,7 \\
\hline Total & 689 & 100 & 78,3 & 164 & 100 & 18,64 & 27 & 100 & 3,07 & 880 & 100 \\
\hline
\end{tabular}

Em relação à morfologia ou tipo de células dos cânceres, o carcinoma foi o mais predominante com 621 casos $(70,57 \%)$, seguido do adenocarcinoma, representado por 241 casos $(27,39 \%)$. Outros tipos morfológicos foram encontrados, representando 18 casos $(2,05 \%)$.

\section{DISCUSSÃO}

A caracterização do perfil sociodemográfico e clínico de mulheres com câncer no trato genital submetidas à radioterapia, objeto deste estudo, permite, prioritariamente, a avaliação de questões sociais e da área da saúde, apresentadas no decorrer desta discussão.

A necessidade do tratamento radioterápico mostra claramente a limitação da atenção precoce aos desvios de saúde, considerando que a maioria dos casos poderia ter sido tratada precocemente, antes mesmo do surgimento dos cânceres, por serem doenças preveníveis e curáveis. Observa-se, diante dos resultados, a necessidade de diagnósticos precoces e incentivos às políticas públicas para prevenção das lesões pré-neoplásicas.

Apesar desta evidência não ser uma novidade no meio científico, ela reafirma que, 
mesmo diante de tantos avanços científicos, a atenção à saúde da mulher, iniciando pela atenção básica, exige providências urgentes (amplamente divulgadas cientificamente e políticas públicas), mas de baixos custos, quando comparadas com os custos da alta complexidade para controle dos cânceres no trato genital.

Neste contexto de doença já avançada, a radioterapia é percebida como grande contribuidora no controle da doença, apesar de seu impacto biopsicossocial às mulheres (alterações gastrointestinais, hematológicas, cutâneas/mucos, fertilidade, sexualidade, nos relacionamentos, ansiedade, medos dentre outros) ${ }^{(4)}$. Para melhores prognósticos, a necessidade de sua indicação também deve ser a mais precoce possível.

A caracterização do perfil mostrou que, com relação à variável idade, assemelhase aos resultados de outros estudos que avaliam o perfil sociodemográfico e clínico de mulheres com câncer de colo do útero. A faixa etária mais predominante encontrada por outros dois estudos realizados no Estado do Espírito Santo e na cidade de Teresina-PI foram, respectivamente, 40 e 59 anos e 50 e 59 anos ${ }^{(7-9)}$. A idade é um fator de risco contribuinte para o desenvolvimento dos cânceres no trato genital. Para o câncer de colo do útero, quanto maior a idade, maior chance de desenvolvimento do câncer. A literatura aponta que esta neoplasia é considerada rara em mulheres até 30 anos e sua incidência aumenta progressivamente até ter seu pico na faixa de 45 a 50 anos de idade ${ }^{(10)}$.

O câncer de ovário ocorre, principalmente, em mulheres pós-menopausa, entre a sexta e sétima décadas de vida(11). Tal evidência também foi encontrada neste estudo. $O$ câncer vaginal ocorre em menos de $1 \%$ dos casos dos cânceres femininos, tendo maior incidência entre 65 e 69 anos, conforme dados divulgados pelo Cancer Research UK ${ }^{(12)}$. Os resultados encontrados no Reino Unido são similares aos encontrados neste estudo. Em relação ao câncer na vulva, os achados diferem dos achados no Reino Unido(12). Em Santa Catarina, a média de idade das mulheres acometidas foi de 55 anos, enquanto no Reino Unido, a maioria dos casos ocorreu entre os 75-85 anos.

O câncer do endométrio representa o câncer ginecológico mais comum nos países desenvolvidos, sendo que sua incidência vem aumentando. As mulheres são diagnosticadas, frequentemente, quando a doença ainda está confinada ao corpo do útero(13). As taxas de incidência, segundo a idade, aumentam acentuadamente a partir dos 50 anos $(90 \%$ dos casos), sendo a ocorrência da maior incidência ocorre na faixa etária de 65-69 anos, e a idade médias das mulheres 63 anos, ${ }^{(14)}$ assemelhando-se aos achados deste estudo.

Quanto à escolaridade, o ensino fundamental incompleto e completo foi o que mais se destacou. Em outro estudo ${ }^{(9)}$ realizado no território nacional, o ensino fundamental também foi o que atingiu o maior percentual (49\%). Em estudo realizado em uma capital do Nordeste ${ }^{(8)} 38,8 \%$ das mulheres tinham ensino fundamental incompleto, em capital da região Sudeste $70,9 \%$ das mulheres tinha até o fundamental incompleto(7). Entretanto, no presente estudo, como pode ser observado nos resultados apresentados, os percentuais em Santa Catarina foram ainda mais elevados. Assim, fica evidente que a baixa escolaridade, associada ao padrão socioeconômico mais carente e às dificuldades de acesso aos serviços de saúde e à educação em saúde, contribuem para o diagnóstico tardio das neoplasias do trato genital.

A raça/cor predominante foi a branca. Isso se dá pelo fato de Santa Catarina ser um Estado com colonização principalmente europeia (espanhóis, italianos, alemães e açorianos), predominantemente com cor de pele branca. Essa realidade difere de estudo brasileiro(9) em que a cor parda foi a mais prevalente com 47,9\% nos casos, e outros dois estudos $^{(7-8)}$ apontam a cor não branca com maior percentual, respectivamente, $76,8 \%$ e $82,1 \%$.

Casada foi o estado conjugal mais frequente identificado entre as mulheres com cânceres, como já apontado nos resultados, reafirmando achados de outros estudo ${ }^{(7-9)}$. Portanto, evidencia-se que na fase da vida classificada como adulta jovem e adulta há maior acometimento de neoplasias malignas no trato genital, e nesta fase da vida também se encontra a maior frequência de mulheres casadas no Brasil, cerca $57,1 \%$ da população 
brasileira acima de 15 anos tem algum tipo de união conjugal(15).

Neste estudo, os estadiamentos com maior percentual encontrado foram o III e o II. Em outros três estudos ${ }^{(7-9)}$ os resultados foram equivalentes. Ressalta-se que o estadiamento auxilia na identificação do comportamento do tumor e da seleção da terapêutica adequada. Quanto mais precoce for o diagnóstico menor o estadiamento, melhor será o prognóstico e o controle da doença.

O principal fator associado ao estadiamento avançado do câncer de colo do útero, maior incidência de casos neste estudo, é a presença de carcinoma de células escamosas, sendo que as mulheres com idades mais elevadas têm sido frequentemente diagnosticadas em estádio avançado, mais do que as jovens. Estima-se que para cada ano adicional na idade, a chance de ter o diagnóstico com carcinoma em estadiamento avançado aumente em $3 \%{ }^{(16)}$.

Cabe destacar que, dentre os cânceres do trato genital, o câncer do colo do útero foi o mais frequente na população estudada, sendo que nos estádios avançados, a radioterapia é uma das terapêuticas de eleição(17). Assim, justifica-se o número de atendimentos no cenário do estudo, relacionado à exclusividade do tratamento por braquiterapia no Estado de Santa Catarina até o ano 2016 ao cenário do estudo.

A procedência das mulheres que mais se destacou foi a proveniente da macrorregião da grande Florianópolis, seguida da macrorregião Sul, Extremo Oeste e Nordeste. O CEPON tem a responsabilidade de prestar atendimento à Grande Florianópolis (que inclui os 22 municípios próximos à cidade de Florianópolis) e ao município de Laguna(18) no que se refere à oferta da braquiterapia, assim, justificam-se os achados.

Outro fator que também influenciou os resultados obtidos foi a incidência das doenças por macrorregiões e a inexistência de braquiterapia de alta taxa de dose fora da Grande Florianópolis entre os anos de 2010-2014. Por este motivo, o CEPON era responsável pela administração da terapêutica braquiterápica para todas as mulheres de todas as macrorregiões, elevando assim o número de seu atendimento. As mulheres que necessitaram de outras terapêuticas e de teleterapia puderam realizar o atendimento oncológico em outras macrorregiões. Todavia, a maioria das mulheres com câncer do colo do útero invasivo, que também foi o mais frequente dos casos deste estudo, é submetida à braquiterapia por alta taxa de dose. O atendimento distribuído nas macrorregiões descentraliza o atendimento, seguindo assim um princípio do Sistema Único de Saúde.

Otipo celular mais encontrado no estudo foi o carcinoma, seguido de adenocarcinoma. Este resultado é similar a outros estudos, $88,0 \%$ dos casos corresponderam a carcinoma, enquanto $10,6 \%$ a adenocarcinoma ${ }^{(9)}, 87 \%$ carcinoma e $11,1 \%$ adenocarcinoma $^{(7)}$ e $51,1 \%$ de carcinoma ${ }^{(8)}$. Registra-se que o carcinoma de células escamosas é o mais comum dos cânceres do colo do útero ${ }^{(7)}$.

A incidência dos casos dos cânceres de colo do útero, dentre os cânceres do trato genital, mostra o quanto esta neoplasia continua acometendo muitas mulheres. Apesar dos avanços na área da saúde e das políticas públicas específicas para o controle da doença, ainda há muito a ser realizado para redução dos números de mulheres que recebem o diagnóstico de câncer no colo do útero. A melhoria da formação dos profissionais é um caminho, bem como a educação permanente, a qualificação dos exames diagnósticos, e a educação da população para controle dos fatores de risco e detecção precoce. Outra estratégia, em longo prazo, relaciona-se a educação em saúde para meninas e meninos serem vacinados para prevenir as infecções por repetição do Vírus do Papiloma Humano (HPV).

Portanto, considera-se essencial que os profissionais envolvidos no diagnóstico dos cânceres do trato genital trabalhem em consonância, para garantir o diagnóstico precoce e a melhor qualidade e acurácia dos exames clínicos, laboratoriais e de imagem que permitem esse diagnóstico. Neste contexto, os profissionais de enfermagem, como articuladores do processo de cuidado, podem contribuir significativamente nos diversos níveis de atenção 
para a melhor qualidade de vida e saúde da mulher ${ }^{(19)}$.

\section{CONCLUSÃO}

Em relação ao perfil sociodemográfico das mulheres com câncer no trato genital submetidas à radioterapia no CEPON entre 2010 a 2014, destaca-se que a maioria estudou até o ensino fundamental, 558 (63,41\%), e estava na faixa etária dos 40 aos 49 anos, 204 $(23,18 \%)$. A maior taxa de prevalência dos casos - entre 60 a 69 anos - com 82,28\% dos casos para cada 100.000 mulheres.

Sobre o perfil clínico, a maior topografia relaciona-se ao câncer de colo do útero, 695 casos (78,97\%); seguida do câncer de corpo do útero, 166 casos $(18,87 \%)$; destacandose no corpo do útero o endométrio com 159 casos (18,06\%). Quanto ao estadiamento, os estádios III, com 315 (35,8\%), seguido do II, 293 (33,3\%), foram os mais frequentes, mostrando o diagnóstico tardio das doenças e a fragilidade do sistema de saúde para prevenção e diagnóstico precoce.

Diante dos achados, fica evidente a importância do controle do câncer do colo do útero nas fases pré-neoplásicas e nas fases iniciais da doença, com acompanhamento das mulheres para detecção precoce das outras neoplasias do trato genital. Deve-se considerar as sequelas das doenças para mulheres e a repercussão dessas, interferindo, muitas vezes, na qualidade de vida pessoal e social.

Como estratégia de controle, destaca-se a implementação de políticas públicas já existentes, a educação permanente dos profissionais e a educação em saúde das mulheres. Neste contexto, os achados deste estudo podem contribuir para avaliação e replanejamento das ações preventivas, de detecção precoce e de atenção na alta complexidade. E ainda, fica evidente a importância do incentivo e oportunização do ensino e informação.

As limitações do presente estudo referem-se ao período relativamente curto para a construção de uma série histórica.

\section{REFERÊNCIAS}

1. Ervik M, Lam F, Ferlay J, Mery L, Soerjomataram I, Bray F. Cancer Today. Lyon, France: International Agency for Researchon Cancer. [Internet]. 2018 [acesso em 06 nov 2018]. Disponível em: http://gco.iarc.fr/ today/data/factsheets/cancers/23-Cervix-Uteri-fact-sheet.pdf.

2. Instituto Nacional de Câncer (INCA). Estimativa 2018: Incidência de Câncer no Brasil. Rio de Janeiro: INCA; 2018.

3. Marta GN, Hanna SA, Silva JLF, Carvalho HA. Câncer de próstata localizado: teleterapia, braquiterapia ou prostatectomia radical? Diagn Tratamento. [Internet]. 2012 [acesso em 13 maio 2017]; 17(2) Disponível em: http://files.bvs.br/upload/S/1413-9979/2012/v17n2/a3027.pdf.

4. Sales CP, Carvalho HA, Taverna KC, Pastorello BF, Rubo RA, Borgonovi AF, et al. Evaluation of different magnetic resonance imaging contrast materials to be used as dummy markers in image-guided brachytherapy for gynecologic malignancies. Radiol Bras. [Internet]. 2016 [acesso em 15 mar 2018]; 49(3). Disponível em: http://dx.doi.org/10.1590/0100-3984.2015.0004.

5. Instituto Nacional de Câncer José Alencar Gomes da Silva (INCA). Informações dos Registros Hospitalares de Câncer como estratégia de transformação: perfil do Instituto Nacional José Alencar Gomes da Silva. Rio de Janeiros: INCA; 2012.

6. Andrade CT, Magedanz AMPCB, Escobosa DM, Tomaz WM, Santinho CS, Lopes TO, et al. A importância de uma base de dados na gestão de serviços de saúde. Einstein. [Internet]. 2012 [acesso em 15 jan 2018]; 
10(3). Disponível em: http://www.scielo.br/pdf/eins/v10n3/v10n3a18.pdf.

7. Mascarello KC, Silva NF, Piske MT, Viana KCG, Zandonade E, Amorim MHC. Perfil Sociodemográfico e Clínico de Mulheres com Câncer do Colo do Útero Associado ao Estadiamento Inicial. Rev. bras. cancerol. [Internet] 2012 [acesso em 15 mar 2018]; 58(3). Disponível em: http://www.inca.gov.br/rbc/n 58/v03/ pdf/11 artigo perfil sociodemografico clinico mulheres cancer colo utero associado estadiamento inicial.pdf.

8. Ribeiro JF, Silva ARV, Campelo V, Santos SLD, Coêlho DMM. Perfil sociodemográfico e clínico de mulheres com câncer do colo do útero em uma cidade do nordeste. Rev Eletr Gestão Saúde. [Internet]. 2015 [acesso em 12 jan 2018]; 6(2). Disponível em: http://periodicos.unb.br/index.php/rgs/article/view/13799/9733.

9. Thuler LCS, Bergmann A, Casado L. Perfil das Pacientes com Câncer do Colo do Útero no Brasil, 20002009: estudo de base secundária. Rev. bras. cancerol. [Internet]. 2012 [acesso em 25 maio 2017]; 58(3). Disponível em: http://www1.inca.gov.br/rbc/n 58/v03/pdf/04 artigo perfil pacientes cancer colo utero brasil 20002009 estudo base secundaria.pdf.

10. Speck NMG, Pinheiro JS, Pereira ER, Rodrigues D, Focchi GRA, Ribalta JCL. Cervical cancer screening in young and elderly women of the Xingu Indigenous Park: evaluation of the recommended screening age group in Brazil. Einstein. [Internet]. 2015 [acesso em 15 mar 2018]; 13(1). Disponível em: http://dx. doi. org/10.1590/S1679-45082015AO3222.

11. Reid BM, Permuth JB, Sellers TA.Epidemiology of ovarian cancer: a review. Cancer Biol Med. [Internet]. 2017 [acesso em 12 jan 2018]; 14(1). Disponível em: http://dx.doi.org/10.20892/j.issn.2095-3941.2016.0084.

12. Cancer Research UK. Uterine cancer incidenc estatistics. [Internet]. 2016 [acesso em 25 maio 2017]. Disponível em: http://www.cancerresearchuk.org/health-professional/cancer-statistics/statistics-by-cancertype/uterine-cancer/incidence\#heading-One.

13. Morice P, Leary A, Creutzberg C, Abu-Rustum N, Darai E. Endometrial cancer. Lancet. [Internet]. 2016 [acesso em 5 maio 2017]; 387(10023). Disponível em: https://doi.org/10.1016/S0140-6736(15)00130-0.

14. Braun MM, Overbeek-Wagner EA, Grumbo RJ. Diagnosis and managment of endometrial câncer. Am Fam Physician. [Internet]. 2016 [acesso em 12 jan 2018]; 93(6). Disponível em: https://www.aafp.org/ afp/2016/0315/p468.pdf.

15. Instituto Brasileiro de Geografia e Estatística (IBGE). Ministério do Planejamento Orçamento e Gestão. Brasil em números. Rio de Janeiro: IBGE; 2014.

16. Thuler LCS, Aguiar SS, Bergmann A. Determinantes do diagnóstico em estadio avançado do câncer do colo do útero no Brasil. Rev. bras. ginecol. obstet. [Internet]. 2014 [acesso em 25 maio 2017]; 36(6). Disponível em: http://dx.doi.org/10.1590/S0100-720320140005010.

17. Tsunoda AT, Andrade CEMC, Vieira MA, Reis R. Laparoscopy in uterine cervical cancer. Current state and literature review. Rev. Col. Bras. Cir. [Internet]. 2015 [acesso em 15 mar 2018]; 42(5). Disponível em: http://dx.doi.org/10.1590/0100-69912015005014.

18. Secretaria de Estado da Saúde (SC). Plano de ação da rede de atenção a saúde das pessoas com câncer em Santa Catarina. [Internet]. 2016 [acesso em 26 maio 2017]. Disponível em: http://portalses.saude.sc.gov. br/index.php?option=com docman\&task=doc download\&gid=10183\&ltemid=82.

19. Lima TM, Lessa PRA, Freitas LV, Teles LMR, Aquino PS, Damasceno AKC, et al. Análise da capacidade diagnóstica dos exames preventivos do câncer de colo uterino. Acta paul. enferm. [Internet]. 2012 [acesso em 12 jan 2018]; 25(5). Disponível em: http://dx.doi.org/10.1590/S0103-21002012000500005. 
Autor Correspondente:

Luciana Martins da Rosa

Universidade Federal de Santa Catarina

Campus Reitor João David Ferreira Lima, s/n - 88040-900 - Florianópolis, SC, Brasil

E-mail: luciana.m.rosa@ufsc.br

Contribuição dos autores:

Elaboração e revisão crítica do conteúdo intelectual do estudo - VR, MMM, HIVMF, EBD

Responsável por todos os aspectos do estudo, assegurando as questões de precisão ou integridade de qualquer parte do estudo - AALS, LMR, SDS 